\title{
Pancreatic Intraductal Papillary Mucinous Neoplasm With Elevated Pre-Operative Cystic Carcinoembryonic Antigen Level: A Histopathologic Correlation
}

\author{
Cameron Beech ${ }^{\mathrm{a}}$, Mollie Freedman-Weiss ${ }^{\mathrm{b}}$, Ronald Salem ${ }^{\mathrm{b}}$, \\ Dhanpat Jain ${ }^{\mathrm{a}}$, Xuchen Zhang, c
}

\begin{abstract}
Background: To study the relationship between carcinoembryonic antigen (CEA) level, intraductal papillary mucinous neoplasm (IPMN) subtype, and the presence of invasive carcinoma.

Methods: Cystic CEA level and the following pathologic variables: subtypes of IPMN, size of cystic lesion, presence of dysplasia or carcinoma, and main or branch duct involvement from 45 IPMN cases were analyzed.

Results: There was a significant correlation between pre-operative cystic fluid CEA level and the intensity of luminal CEA staining. However, there was no correlation between CEA level and cystic mucinous secretions or mucinous epithelial cytoplasm CEA staining, mucin glycoprotein expression, size of lesion, grade of dysplasia or presence of invasive carcinoma. CEA level was neither sensitive nor specific for the presence of invasive carcinoma.

Conclusions: Cystic CEA level may not be a reliable determinant of the presence or absence of invasive carcinoma in IPMNs, and its use to assess risk of malignancy may be limited.
\end{abstract}

Keywords: Intraductal papillary mucinous neoplasm; Mucin glycoprotein; Pancreas; Carcinoembryonic antigen

\section{Introduction}

Intraductal papillary mucinous neoplasm (IPMN) is a substantial subclass of pancreatic pathology, representing $20-50 \%$ of

Manuscript submitted July 7, 2019, accepted July 16, 2019

aDepartment of Pathology, Yale University School of Medicine, New Haven, CT, USA

bSection of Surgical Oncology, Yale University School of Medicine, New Haven, CT, USA

${ }^{\mathrm{c} C}$ Corresponding Author: Xuchen Zhang, Department of Pathology, Yale University School of Medicine, 310 Cedar Street, PO Box 208023, New Haven, CT 06510, USA. Email: Xuchen.zhang@yale.edu

doi: https://doi.org/10.14740/gr1201 cystic pancreatic neoplasms [1-3]. The incidence and subsequent resection of IPMN have increased over the last 20 years, partially explained by the increased utilization of high-resolution diagnostic imaging [4]. IPMN is thought to represent a precursor lesion to pancreatic adenocarcinoma due to its association with invasive carcinoma [5]. Due to the increased recognition of pancreatic cystic lesions, adequately stratifying patients based on their malignancy risk is increasingly important; particularly so, when deciding between surgical intervention and active surveillance.

Several pathologic variables have been implicated in the risk evaluation of malignancy for IPMN including branch duct vs. main duct involvement and presence or absence of a cystic mural nodule [6-8]. Four main histologic subtypes of IPMN have been described: gastric, intestinal, pancreaticobiliary and oncocytic. These subtypes differ in their prognosis and their risk for dysplasia and/or invasive carcinoma [9-11]. Mucin glycoprotein expression in IMPN epithelial cells within the cyst lining is routinely used to help characterize these IPMN subtypes by immunohistochemistry [12]. Although these biomarkers are useful in helping distinguish individual IPMN subtypes, this immunohistochemical assessment is often only possible after resection.

In an attempt to identify pancreatic cystic lesions with an increased risk of associated invasive adenocarcinoma, carcinoembryonic antigen (CEA) level has emerged over the last few decades as a tool to assess pancreatic cystic fluid pre-operatively. During the diagnostic workup for pancreatic cysts, CEA is often utilized to distinguish those pancreatic cystic lesions that are likely to be mucinous [13-16]. In addition to CEA being used to stratify mucinous vs. non-mucinous lesions, several studies have attempted to correlate pancreatic cyst fluid CEA level and the presence of invasive carcinoma, with discordant results [17, 18]. As it stands, the pathologic correlate of elevated CEA within cyst fluid remains unclear. Moreover, the relationship between CEA and IPMN subtypes has not been sufficiently assessed.

Therefore, the aims of this study were to correlate pre-operative cyst fluid CEA level with the histopathologic features and mucin glycoprotein expression of IPMN after pancreatic resection.

\section{Materials and Methods}

The study was approved by appropriate institutional review 


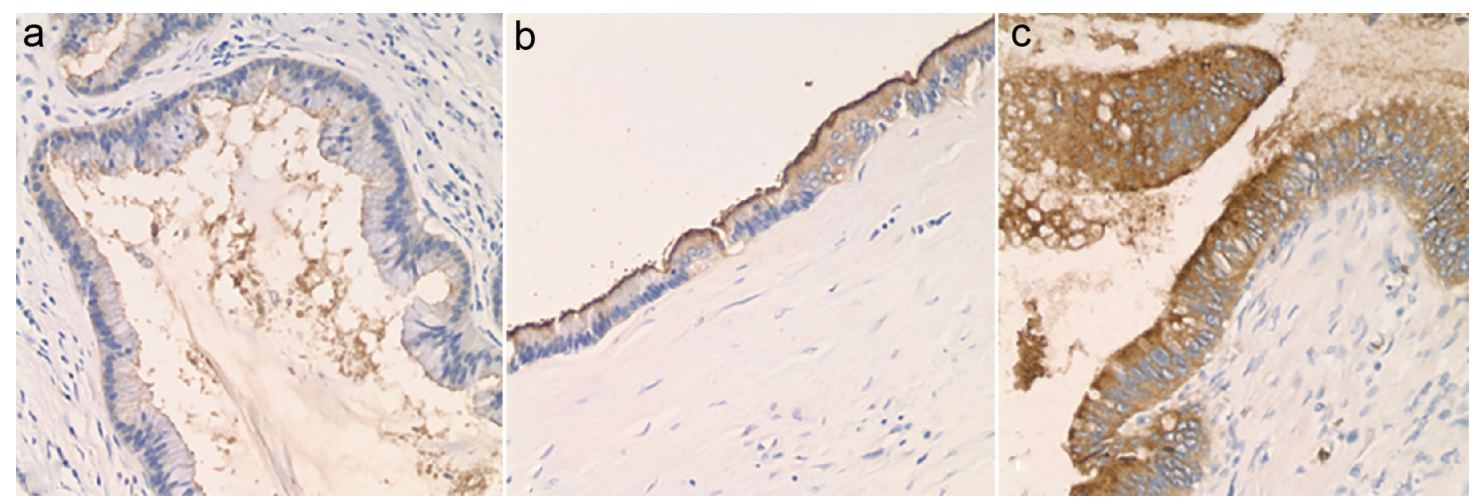

Figure 1. Luminal staining for CEA, assessed by immunohistochemistry and graded as 0 to $3+$. (a) Luminal CEA: $1+(\times 200)$. (b) Luminal CEA: $2+(\times 200)$. (c) Luminal CEA: $3+(\times 200)$.

board, and followed the ethical compliance with human study. Forty-five cases of pancreatic resection for IPMN between the years 2003 - 2014 were retrospectively reviewed. Pathologic variables of the resected IPMNs included in the analysis were: the subtype of IPMN, pathologic size of cystic lesion, pre-resection pancreatic cyst fluid CEA level $(\mathrm{ng} / \mathrm{mL})$, presence or absence of dysplasia (low or high grade), presence or absence of invasive carcinoma, and main duct or branch duct involvement. Pancreatic cyst fluid CEA was assessed by enzyme immunoassay (AccuCea Redpath Integrated Pathology, now Interpace Diagnostics). A cut-off for elevated CEA, indicating a most likely mucinous IPMN, was set at $>192 \mathrm{ng} / \mathrm{mL}$, per the test manufactures recommendations. Due to an insufficient number of cases with cyst fluid amylase measurements, amylase measurements were not included in the study.

Protein expression was assessed via immunohistochemistry (IHC) for circulating anti-mucin (MUC) 1, MUC2, MUC5, MUC6, polyclonal CEA (CEA-P), and CDX2 on each case. IHC positivity for each marker was scored based on intensity of staining, from 0 (no staining) to $3+$ (strong staining) (Fig. 1). As a negative control, four cases of serous cystadenoma were stained for CEA-P. Immunohistochemical positivity was assessed within the following locations: intra-cystic secretions, luminal surface and cytoplasm of neoplastic epithelium and the adjacent non-lesional pancreatic tissues. Pathologic variables and IHC findings were compared to pre-operative pancreatic cyst CEA level. Descriptive statistics and bivariate correlation analysis were carried out using IBM SPSS (version 1.0.0-1058). In attempt to control for random variation, outliers were identified and removed when present at 1.5 interquartile ranges (IQRs) below the first quartile or above the third quartile.

\section{Results}

\section{Descriptive statistics of cohort}

After removing outliers, a total of 40 resection specimens were assessed, including three major IPMN subtypes: pancreaticobiliary $(n=6)$, gastric $(n=30)$, and intestinal $(n=4)$. Five of the resection specimens had concomitant invasive adenocarcinoma (pancreaticobiliary $(\mathrm{n}=2)$, gastric $(\mathrm{n}=2)$, and intestinal a

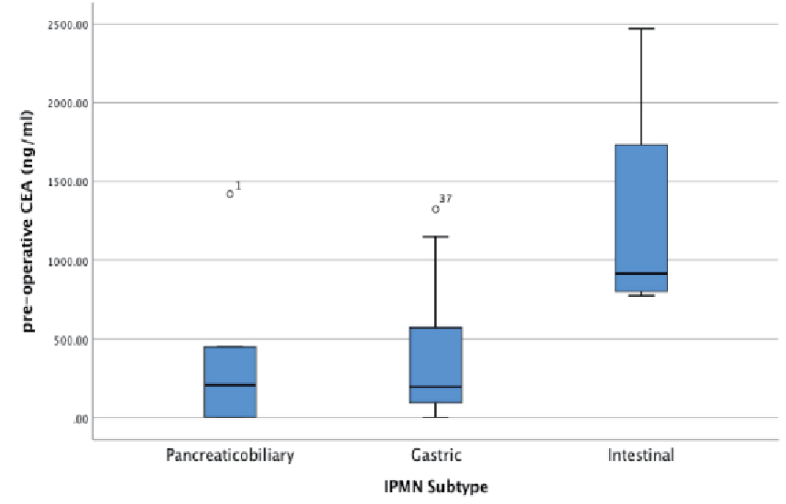

b

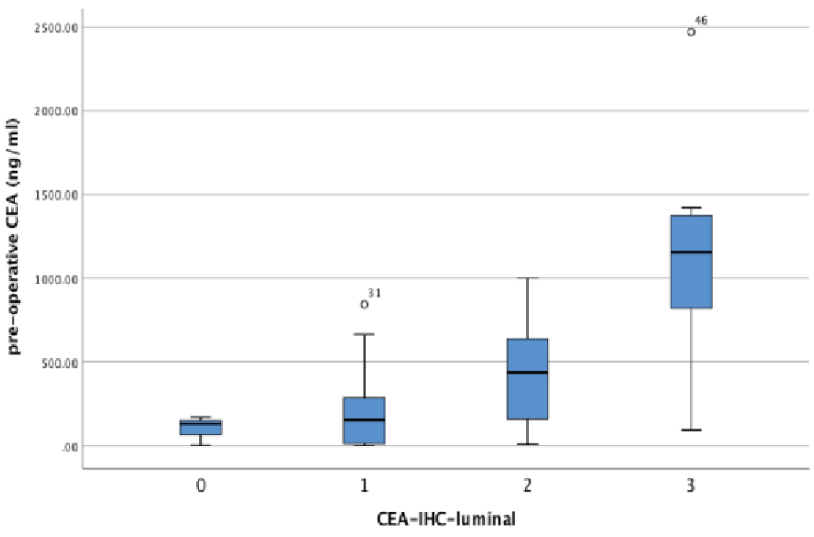

Figure 2. (a) Box and whisker plot of pre-operative IPMN cyst fluid CEA level (ng/mL) vs. IPMN subtypes. (b) Box and whisker plot of pre-operative IPMN cyst fluid CEA level $(\mathrm{ng} / \mathrm{mL})$ vs. CEA luminal staining assessed by immunohistochemistry of resected IPMNs. 
Table 1. Pearson Correlation Between Pre-Operative Pancreatic Cyst Fluid CEA Level and CEA Immunohistochemical Staining Intensity

\begin{tabular}{lll}
\hline \multirow{2}{*}{ CEA-P-IPMN lumenal } & Pearson correlation & CEA level \\
& Sig. (two-tailed) & 0.611 \\
& $\mathrm{~N}$ & $0.000^{*}$ \\
CEA-P-IPMN cytoplasm & Pearson correlation & 0.099 \\
& Sig. (two-tailed) & 0.541 \\
& N & 40 \\
CEA-P-IPMN secretion & Pearson correlation & 0.028 \\
& Sig. (two-tailed) & 0.861 \\
& $\mathrm{~N}$ & 40 \\
\hline
\end{tabular}

${ }^{*}$ Correlation is significant at the 0.05 level (two-tailed).

$(\mathrm{n}=1)$ subtypes $)$. No oncocytic subtypes were identified in this study.

\section{Correlation studies: CEA and histopathologic variables}

Amongst the IPMN subtypes, the intestinal subtype had the largest median cyst fluid CEA level (median: $914.50 \mathrm{ng} / \mathrm{mL}$; mean: $1268.00 \pm 403.21 \mathrm{ng} / \mathrm{mL}$ ), followed by pancreaticobiliary subtype (median: $207.00 \mathrm{ng} / \mathrm{mL}$; mean: $381.28 \pm 223.90$ $\mathrm{ng} / \mathrm{mL}$ ) and gastric subtype (median: $193.00 \mathrm{ng} / \mathrm{mL}$; mean: $368.99 \pm 65.91 \mathrm{ng} / \mathrm{mL}$ ) (Fig. 2a). In addition, there was a statistically significant correlation between pre-operative cyst fluid CEA level and the intensity of luminal CEA staining (Pearson $\mathrm{r}=0.611 ; \mathrm{P}=0.00003$ ) (Fig. 2b, Table 1). However, no correlation between pre-operative cyst fluid CEA level and CEA IHC staining within cystic mucinous secretions or mucinous epithelial cytoplasm was identified (Table 1). Similarly, there was no correlation between pre-operative cyst fluid CEA level and other IHC markers (MUC1, 2, 5, or 6) (Table 2), size of lesion (Fig. 3, Table 3), grade of dysplasia, or presence of invasive carcinoma (Table 3). As expected, no staining for CEA-P was identified within the four cases of serous cystadenoma. Pre-operative pancreatic cyst CEA was neither sensitive nor specific for the presence of invasive carcinoma, with an area under the curve of 0.449 (Fig. 4). Intensity of CDX2 nuclear staining correlated with pre-operative cyst fluid CEA (Pearson $\mathrm{r}=0.343 ; \mathrm{P}=0.03)$ (Table 3).

\section{Discussion}

Pancreatic cyst lesions are frequently found incidentally during abdominal imaging for non-pancreatic symptomatology, comprising approximately $2.4-13.5 \%$ of patients with no history of pancreatic disease $[19,20]$. The rate of pancreatic cyst disease appears to increase with age with several imaging and autopsy studies show an increased prevalence after the age of 70 years [20, 21]. Management for pancreatic cystic lesions depends on the risk of malignancy and/or progression towards
Table 2. Pearson Correlation Between Pre-Operative Pancreatic Cyst Fluid CEA Level and MUC1, MUC2, MUC5, MUC6 Immunohistochemical Staining Intensity

\begin{tabular}{|c|c|c|}
\hline & & CEA level \\
\hline \multirow[t]{3}{*}{ MUC1-IPMN lumenal } & Pearson correlation & -0.137 \\
\hline & Sig. (two-tailed) & 0.477 \\
\hline & $\mathrm{N}$ & 29 \\
\hline \multirow[t]{3}{*}{ MUC1-IPMN cytoplasm } & Pearson correlation & -0.103 \\
\hline & Sig. (two-tailed) & 0.594 \\
\hline & $\mathrm{N}$ & 29 \\
\hline \multirow[t]{3}{*}{ MUC1-IPMN secretion } & Pearson correlation & -0.076 \\
\hline & Sig. (two-tailed) & 0.696 \\
\hline & $\mathrm{N}$ & 29 \\
\hline \multirow[t]{3}{*}{ MUC2-pancrease } & Pearson correlation & -0.082 \\
\hline & Sig. (two-tailed) & 0.616 \\
\hline & $\mathrm{N}$ & 40 \\
\hline \multirow[t]{3}{*}{ MUC2-IPMN cytoplasm } & Pearson correlation & 0.259 \\
\hline & Sig. (two-tailed) & 0.107 \\
\hline & $\mathrm{N}$ & 40 \\
\hline \multirow[t]{3}{*}{ MUC5-IPMN lumenal } & Pearson correlation & 0.000 \\
\hline & Sig. (two-tailed) & 0.998 \\
\hline & $\mathrm{N}$ & 40 \\
\hline \multirow[t]{3}{*}{ MUC5-IPMN cytoplasm } & Pearson correlation & -0.039 \\
\hline & Sig. (two-tailed) & 0.812 \\
\hline & $\mathrm{N}$ & 40 \\
\hline \multirow[t]{3}{*}{ MUC5-IPMN secretion } & Pearson correlation & 0.038 \\
\hline & Sig. (two-tailed) & 0.818 \\
\hline & $\mathrm{N}$ & 40 \\
\hline \multirow[t]{3}{*}{ MUC6-IPMN lumenal } & Pearson correlation & -0.126 \\
\hline & Sig. (two-tailed) & 0.507 \\
\hline & $\mathrm{N}$ & 30 \\
\hline \multirow[t]{3}{*}{ MUC6-IPMN cytoplasm } & Pearson correlation & 0.259 \\
\hline & Sig. (two-tailed) & 0.167 \\
\hline & $\mathrm{N}$ & 30 \\
\hline \multirow[t]{3}{*}{ MUC6-IPMN secretion } & Pearson correlation & -0.174 \\
\hline & Sig. (two-tailed) & 0.357 \\
\hline & $\mathrm{N}$ & 30 \\
\hline \multirow[t]{3}{*}{ MUC6-pancrease } & Pearson correlation & -0.105 \\
\hline & Sig. (two-tailed) & 0.582 \\
\hline & $\mathrm{N}$ & 30 \\
\hline
\end{tabular}

invasive carcinoma. Generally, pancreatic cysts can be broadly categorized as neoplastic or non-neoplastic. Neoplastic cysts are further broadly classified as mucinous and nonmucinous cysts [22].

CEA is a useful biomarker for helping to distinguish mucinous from non-mucinous pancreatic cysts. In this study, pre- 


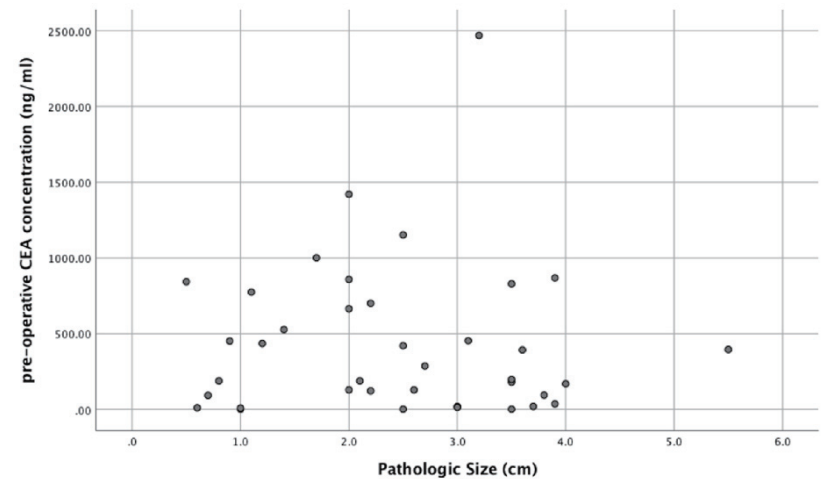

Figure 3. Pre-operative pancreatic cyst fluid CEA level and corresponding tumor size $(\mathrm{cm})$.

operative pancreatic cyst fluid CEA level positively correlated with the intensity of luminal CEA staining in the neoplastic epithelium lining the pancreatic cysts; this is consistent with prior studies suggesting that CEA is primarily expressed on the apical surface of the gastrointestinal epithelium [23]. Interestingly, we did not find association of pre-operative cyst CEA levels with CEA immunointensity in cyst secretion. It is possible there were pre-analytic variable that might impact immunointensity but not impact aspirate fluid measurements such as ischemia time and formalin fixation. It is also possible that CEA as mentioned earlier is expressed on the IPMN epithelial apical surface, may be more stable when it is cell bound rather than within secretions. The intestinal IPMN subtype had the highest cyst fluid CEA level, differing from prior studies suggesting gastric subtypes have the highest CEA levels [24]. Similar to what has been previously described in our study, there was no correlation between pre-operative cyst fluid CEA level and the presence of dysplasia or invasive carcinoma [18, 25]. Other molecular tools to help stratify pancreatic cysts based on malignancy are being explored, but their utility in routine clinical practice remains to be demonstrated [26].

Mucins are heavily glycosylated high molecular weight glycoproteins, which are linked to a protein backbone within

Table 3. Pearson Correlation Between Pre-Operative Pancreatic Cyst Fluid CEA Level and Tumor Size, Presence of Invasive Carcinoma and CDX2 Nuclear Staining Within IPMNs

\begin{tabular}{lll}
\hline & & CEA level \\
\hline Tumor size $(\mathrm{cm})$ & Pearson correlation & -0.015 \\
& Sig. (two-tailed) & 0.930 \\
Carcinoma & N & 38 \\
& Pearson correlation & 0.070 \\
& Sig. (two-tailed) & 0.668 \\
CDX2-nuclear & N & 40 \\
& Pearson correlation & $0.343^{*}$ \\
& Sig. (two-tailed) & 0.030 \\
& N & 40 \\
\hline
\end{tabular}

${ }^{*}$ Correlation is significant at the 0.05 level (two-tailed).

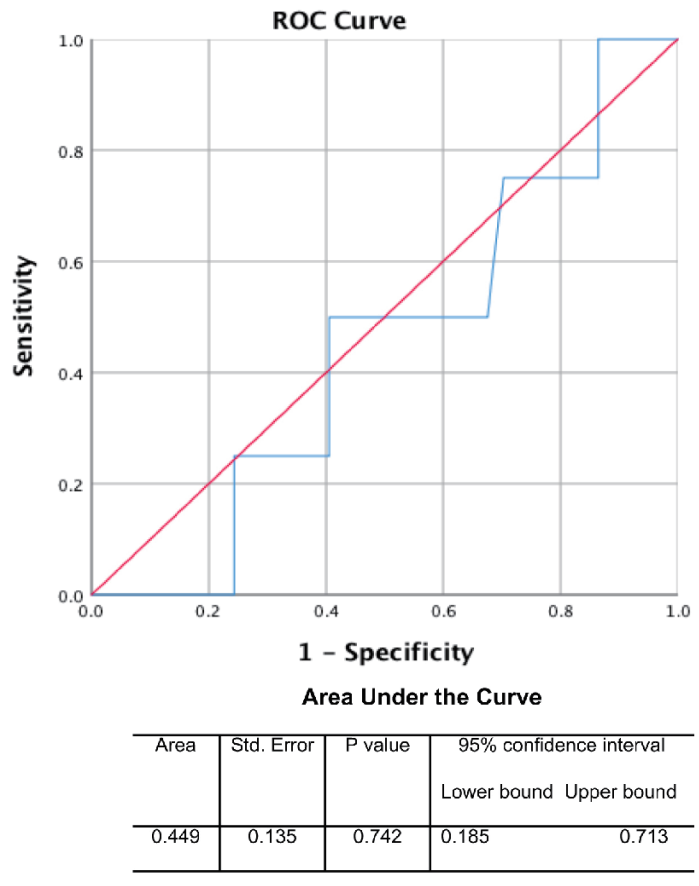

Figure 4. Receiver operator characteristic (ROC) curve of pre-operative CEA cyst fluid level for invasive carcinoma.

epithelial cytoplasmic membranes via serine and threonine residues [27]. Immunohistochemical analysis for various mucin glycoproteins has revealed predictable patterns of mucin glycoprotein expression across both IPMN subtypes and within invasive pancreatic ductal adenocarcinoma [28-30]. MUC1 is a membrane associated mucin which has predominantly increased expression within pancreaticobiliary IPMN subtypes and is also over-expressed within invasive carcinoma. MUC2 is a gel forming mucin, which, along with CDX-2, is predominantly expressed within intestinal IPMN subtypes and rarely detected within invasive carcinoma. MUC5 and MUC6 are gel-forming mucins as well, which are expressed frequently within gastric, intestinal, and oncocytic IPMN subtypes, as well has frequently within invasive carcinoma $[9,31,32]$. In this study, no correlation was identified between mucin glycoprotein expression and pre-operative CEA levels, suggesting that although CEA is elevated within mucinous lesions, no discrete correlation was found to exist with the mucin glycoproteins analyzed.

One of the limitations of our study includes the absence of sufficiently available $K R A S$ mutations status amongst our cases to compare pre-operative cyst fluid level with $K R A S$ and GNAS mutation status. Also, the number of invasive adenocarcinoma $(\mathrm{n}=5)$ in our cohort was limited and therefore may not be entirely representative of the relationship between preoperative CEA level and presence of invasive carcinoma. Further, no oncocytic IPMN subtypes were identified in this study, thus a relationship between pre-operative cyst fluid CEA level and mucin glycoprotein expression could not be assessed in that particular subtype.

Although pre-operative CEA level remains one tool for assessing for the presence of mucinous cystic pancreatic lesions, 
this study attempts to highlight some of the challenges in its utility as a single marker of invasive carcinoma. In addition, to the best of our knowledge, this is the first study comparing mucin glycoprotein expression across subtypes of IPMN to cystic CEA level; and our result was that the mucin glycoprotein expression did not correlate with pre-operative cyst fluid CEA level. The rationale by using CEA levels for undertaking resection of a cystic lesion in pancreas needs to be evaluated more critically.

\section{Acknowledgments}

None to declare.

\section{Financial Disclosure}

None to declare.

\section{Conflict of Interest}

The authors have no conflicts of interest to disclose.

\section{Informed Consent}

Informed consent was waived by the Institutional Review Boards of Yale University due to the retrospective nature of this study.

\section{Author Contributions}

CB collected the data and drafted the manuscript; MFW collected the data and critically reviewed the manuscript; RS, DJ and XZ contributed to the study design and critically reviewed the manuscript.

\section{References}

1. Kosmahl M, Pauser U, Peters K, Sipos B, Luttges J, Kremer B, Kloppel G. Cystic neoplasms of the pancreas and tumor-like lesions with cystic features: a review of 418 cases and a classification proposal. Virchows Arch. 2004;445(2):168-178.

2. Allen PJ, D'Angelica M, Gonen M, Jaques DP, Coit DG, Jarnagin WR, DeMatteo R, et al. A selective approach to the resection of cystic lesions of the pancreas: results from 539 consecutive patients. Ann Surg. 2006;244(4):572582.

3. Lee CJ, Scheiman J, Anderson MA, Hines OJ, Reber HA, Farrell J, Kochman ML, et al. Risk of malignancy in resected cystic tumors of the pancreas $<$ or $=3 \mathrm{~cm}$ in size: is it safe to observe asymptomatic patients? A multi-institutional report. J Gastrointest Surg. 2008;12(2):234-242.
4. Sohn TA, Yeo CJ, Cameron JL, Hruban RH, Fukushima $\mathrm{N}$, Campbell KA, Lillemoe KD. Intraductal papillary mucinous neoplasms of the pancreas: an updated experience. Ann Surg. 2004;239(6):788-797; discussion 797-789.

5. Adsay NV FN, Furukawa T, Hruban RH, Klimstra DS $\mathrm{KG}$, et al. Intraductal neoplasms of the pancreas. Lyon, France: WHO; 2010. pp 304-313.

6. Terris B, Ponsot P, Paye F, Hammel P, Sauvanet A, Molas $G$, Bernades $P$, et al. Intraductal papillary mucinous tumors of the pancreas confined to secondary ducts show less aggressive pathologic features as compared with those involving the main pancreatic duct. Am J Surg Pathol. 2000;24(10):1372-1377.

7. Bernard P, Scoazec JY, Joubert M, Kahn X, Le Borgne J, Berger F, Partensky C. Intraductal papillary-mucinous tumors of the pancreas: predictive criteria of malignancy according to pathological examination of 53 cases. Arch Surg. 2002;137(11):1274-1278.

8. Rodriguez JR, Salvia R, Crippa S, Warshaw AL, Bassi C, Falconi M, Thayer SP, et al. Branch-duct intraductal papillary mucinous neoplasms: observations in 145 patients who underwent resection. Gastroenterology. 2007;133(1):72-79; quiz 309-310.

9. Furukawa T, Kloppel G, Volkan Adsay N, Albores-Saavedra J, Fukushima N, Horii A, Hruban RH, et al. Classification of types of intraductal papillary-mucinous neoplasm of the pancreas: a consensus study. Virchows Arch. 2005;447(5):794-799.

10. Sadakari Y, Ohuchida K, Nakata K, Ohtsuka T, Aishima $\mathrm{S}$, Takahata S, Nakamura $\mathrm{M}$, et al. Invasive carcinoma derived from the nonintestinal type intraductal papillary mucinous neoplasm of the pancreas has a poorer prognosis than that derived from the intestinal type. Surgery. 2010;147(6):812-817.

11. Mino-Kenudson M, Fernandez-del Castillo C, Baba Y, Valsangkar NP, Liss AS, Hsu M, Correa-Gallego C, et al. Prognosis of invasive intraductal papillary mucinous neoplasm depends on histological and precursor epithelial subtypes. Gut. 2011;60(12):1712-1720.

12. Tanaka M. Thirty years of experience with intraductal papillary mucinous neoplasm of the pancreas: from discovery to international consensus. Digestion. 2014;90(4):265-272.

13. Hammel P, Levy P, Voitot H, Levy M, Vilgrain V, Zins M, Flejou JF, et al. Preoperative cyst fluid analysis is useful for the differential diagnosis of cystic lesions of the pancreas. Gastroenterology. 1995;108(4):1230-1235.

14. Lewandrowski KB, Southern JF, Pins MR, Compton CC, Warshaw AL. Cyst fluid analysis in the differential diagnosis of pancreatic cysts. A comparison of pseudocysts, serous cystadenomas, mucinous cystic neoplasms, and mucinous cystadenocarcinoma. Ann Surg. 1993;217(1):41-47.

15. Brugge WR, Lewandrowski K, Lee-Lewandrowski E, Centeno BA, Szydlo T, Regan S, del Castillo CF, et al. Diagnosis of pancreatic cystic neoplasms: a report of the cooperative pancreatic cyst study. Gastroenterology. 2004;126(5):1330-1336.

16. Snozek CL, Mascarenhas RC, O'Kane DJ. Use of cyst 
fluid CEA, CA19-9, and amylase for evaluation of pancreatic lesions. Clin Biochem. 2009;42(15):1585-1588.

17. Maire F, Voitot H, Aubert A, Palazzo L, O'Toole D, Couvelard A, Levy $\mathrm{P}$, et al. Intraductal papillary mucinous neoplasms of the pancreas: performance of pancreatic fluid analysis for positive diagnosis and the prediction of malignancy. Am J Gastroenterol. 2008;103(11):2871-2877.

18. Kucera S, Centeno BA, Springett G, Malafa MP, Chen YA, Weber J, Klapman J. Cyst fluid carcinoembryonic antigen level is not predictive of invasive cancer in patients with intraductal papillary mucinous neoplasm of the pancreas. JOP. 2012;13(4):409-413.

19. Lee KS, Sekhar A, Rofsky NM, Pedrosa I. Prevalence of incidental pancreatic cysts in the adult population on MR imaging. Am J Gastroenterol. 2010;105(9):2079-2084.

20. Kimura W, Nagai H, Kuroda A, Muto T, Esaki Y. Analysis of small cystic lesions of the pancreas. Int J Pancreatol. 1995;18(3):197-206.

21. Zhang XM, Mitchell DG, Dohke M, Holland GA, Parker L. Pancreatic cysts: depiction on single-shot fast spinecho MR images. Radiology. 2002;223(2):547-553.

22. Basturk O, Coban I, Adsay NV. Pancreatic cysts: pathologic classification, differential diagnosis, and clinical implications. Arch Pathol Lab Med. 2009;133(3):423-438.

23. Thompson JA. Molecular cloning and expression of carcinoembryonic antigen gene family members. Tumour Biol. 1995;16(1):10-16.

24. Yoon WJ, Daglilar ES, Mino-Kenudson M, MoralesOyarvide V, Pitman MB, Brugge WR. Characterization of epithelial subtypes of intraductal papillary mucinous neoplasm of the pancreas with endoscopic ultrasound and cyst fluid analysis. Endoscopy. 2014;46(12):10711077.

25. Correa-Gallego C, Warshaw AL, Fernandez-del Castillo C. Fluid CEA in IPMNs: A useful test or the flip of a coin? Am J Gastroenterol. 2009;104(3):796-797.

26. Kadayifci A, Al-Haddad M, Atar M, Dewitt JM, Forcione DG, Sherman S, Casey BW, et al. The value of KRAS mutation testing with CEA for the diagnosis of pancreatic mucinous cysts. Endosc Int Open. 2016;4(4):E391-396.

27. Nagata K, Horinouchi M, Saitou M, Higashi M, Nomoto M, Goto M, Yonezawa S. Mucin expression profile in pancreatic cancer and the precursor lesions. J Hepatobiliary Pancreat Surg. 2007;14(3):243-254.

28. Tashiro Y, Yonezawa S, Kim YS, Sato E. Immunohistochemical study of mucin carbohydrates and core proteins in human ovarian tumors. Hum Pathol. 1994;25(4):364372.

29. Yonezawa S, Nakamura A, Horinouchi M, Sato E. The expression of several types of mucin is related to the biological behavior of pancreatic neoplasms. J Hepatobiliary Pancreat Surg. 2002;9(3):328-341.

30. Yonezawa S, Horinouchi M, Osako M, Kubo M, Takao $\mathrm{S}$, Arimura Y, Nagata K, et al. Gene expression of gastric type mucin (MUC5AC) in pancreatic tumors: its relationship with the biological behavior of the tumor. Pathol Int. 1999;49(1):45-54.

31. Moschovis D, Bamias G, Delladetsima I. Mucins in neoplasms of pancreas, ampulla of Vater and biliary system. World J Gastrointest Oncol. 2016;8(10):725-734.

32. Shi C, Hruban RH. Intraductal papillary mucinous neoplasm. Hum Pathol. 2012;43(1):1-16. 